\title{
Some Remarks on the Symplectic Pairing on the Moduli Space of Representations of the Fundamental Group of Surfaces
}

\author{
Krishnamurthi GURUPRASAD*†
}

\section{§0. Introduction}

This work grew out of an attempt to understand a conjectural remark made by Professor Kyoji Saito to the author about a possible link between the Fox-calculus description of the symplectic structure on the moduli space of representations of the fundamental group of surfaces into a Lie group and pairs of mutually dual sets of generators of the fundamental group. In fact in his paper [3], Prof. Kyoji Saito gives an explicit description of the system of dual generators of the fundamental group.

If $S$ is a compact surface of genus $g \geq 2$ and its fundamental group $\pi_{1}(S)$ is given by a presentation $\pi_{1}(S)=\left\langle A_{1}, B_{1}, \ldots, A_{g}, B_{g} \mid \prod_{\imath=1}^{g},\left[A_{\imath}, B_{\imath}\right]=I\right\rangle$ (where $I$ is the identity element), then the dual set of generators of $\pi_{1}(S)$ are defined by

$$
\begin{aligned}
& \alpha_{\imath}=C_{\imath-1} B_{\imath}^{-1} C_{i}^{-1} \\
& \beta_{\imath}=C_{i} A_{\imath}^{-1} C_{t-1}^{-1}
\end{aligned}
$$

for $i=1, \ldots, g$ where $C_{i}=\prod_{j=1}^{i}\left[A_{j}, B_{j}\right]$.

It can be checked that $\prod_{i=1}^{g}\left[\alpha_{\imath}, \beta_{l}\right]=I$ so that $\pi_{1}(S)$ (henceforth simply denoted as $\pi$ ) has another presentation $\pi=\left\langle\alpha_{1}, \beta_{1}, \ldots, \alpha_{g}, \beta_{g} \mid \prod_{\imath=1}^{g}\left[\alpha_{i}, \beta_{l}\right]=I\right\rangle$. Both the presentations are mutually dual since the following holds

$$
\begin{aligned}
& A_{\imath}=\mathscr{C}_{\imath-1} \beta_{\imath}^{-1} \mathscr{C}_{\imath}^{-1} \\
& B_{\imath}=\mathscr{C}_{\imath} \alpha_{\imath}^{-1} \mathscr{C}_{i-1}^{-1}
\end{aligned}
$$

Communicated by K. Saito, March 10, 1997.

1991 Mathematics Subject Classification (s): 58D27, 58F05

* Department of Mathematics. Indian Institute of Science, Bangalore-560012, India

${ }^{\dagger}$ We would like to express our deep sorrow. Our friend Krishnamurthi Guruprasad was involved in a fatal car accident and passed away on January 5, 1998. K. Saito (communicator) 
for $i=1, \ldots, g$ where $\mathscr{C}_{i}=\prod_{j=1}^{i}\left[\alpha_{j}, \beta_{j}\right]$.

Now if $G$ is a compact Lie group together with adjoint invariant inner product on its Lie algebra, then the moduli space $M=\operatorname{Hom}(\pi, \mathscr{G}) / \mathscr{G}$ of conjugacy class of representations of $\pi$ into $G$ admits a natural symplectic structure. We explore in this paper the relationship between the Fox-calculus description of this symplectic structure and the mutually dual presentations of the fundamental group described above. In fact this paper re-interprets the results of [2] in the framework of mutually dual presentations of the fundamental group.

\section{Acknowledgements}

I express my profound gratitude to Prof. Kyoji Saito for suggesting this approach and insightful discussions. His down to earth description of the dual generators of the surface-group in his preprint [3] was of immense use to me. I also express my deep gratitude to the Department of Applied Mathematics, Fukuoka University. Japan for its hospitality and support during my visit in June '96 and more particularly to Prof. Y. Suyama and Prof. T. Takakura. My special thanks to Prof. T. Kitano, Prof. T. Kohno, Prof. Momose and Prof. K. Ono for making my trip to Japan memorable and mathematically fruitful. I thank the referee for his valuable comments.

\section{\$1. Fox-Calculus Description of the Symplectic Structure on the Mloduli Space}

Goldman ([1]) described explicitly the symplectic structure on the moduli space in terms of Fox-calculus. We recall this description in this section.

Let $S$ be a compact surface of genus $g \geq 2$ and $\pi:=\pi_{1}(S)$ be its fundamental group. Let $\pi=\left\langle A_{1}, B_{1}, \ldots, A_{g}, B_{g} \mid \mathbb{I}_{l=1}^{g}\left[A_{i}, B_{i}\right]=I\right\rangle$ be a given presentation where $I$ is the identity element. Let $C_{l}=\prod_{j=1}^{l}\left[A_{j}, B_{j}\right]$ and $R=C_{g}$ so that $R=I$ is the unique relation among the generators of $\pi$.

The anti-automorphism \# on the integral group ring $\mathbb{Z} \pi$ is defined by

$$
\text { \# }\left(\sum n_{l} a_{l}\right)=\sum n_{\imath} a_{l}^{-1} \text { for } n_{l} \in \mathbb{Z} \text { and } a_{l} \in \pi \text {. }
$$

Using notations from Fox calculus $[1, \S 3]$ we have

$$
\begin{aligned}
& \frac{\partial R}{\partial A_{\imath}}=C_{\imath-1}\left(I-A_{\imath} B_{\imath} A_{\imath}^{-1}\right)=C_{\imath-1}-C_{\imath} B_{\imath} \\
& \frac{\partial R}{\partial B_{\imath}}=C_{\imath-1}\left(A_{l}-A_{\imath} B_{\imath} A_{l}^{-1} B_{l}^{-1}\right)=C_{t-1} A_{\imath}-C_{\imath}
\end{aligned}
$$

so that

$$
\begin{aligned}
& \# \frac{\partial R}{\partial A_{\imath}}=C_{\imath-1}^{-1}-B_{\imath}^{-1} C_{\imath}^{-1} \\
& \# \frac{\partial R}{\partial B_{\imath}}=A_{\imath}^{-1} C_{\imath-1}^{-1}-C_{\imath}^{-1} .
\end{aligned}
$$


The tangent space $T_{[\rho]}(\mathcal{M})$ at an equivalence class $[\rho] \in \mathcal{M}$ is identified with the first group cohomology $H^{1}\left(\pi, \mathfrak{H}_{\rho}\right)$ where $\mathfrak{H}_{\rho}$ is the $\pi$-module. with the module structure defined by the composition $\pi \stackrel{\rho}{\rightarrow} G \stackrel{\text { Ad }}{\rightarrow}$ Aut $(\mathfrak{S g})$.

$Z^{1}\left(\pi, \mathfrak{S}_{\rho}\right)=\left\{u: \pi \rightarrow \mathfrak{S}_{\rho} \mid u(x y)=u(x)+\operatorname{Ad}_{\rho}(x)(u(v))\right.$ for $\left.x, y \in \pi\right\}$ is the spece of 1 -cocycles.

The following composition of the cup-product and $\langle$, $\rangle$

$$
Z^{1}\left(\pi, \mathfrak{S}_{\rho}\right) \times Z^{1}\left(\pi, \mathfrak{S}_{\rho}\right) \stackrel{\cup}{\rightarrow} Z^{2}\left(\pi, \mathfrak{S}_{\rho} \otimes \mathfrak{S}_{\rho}\right) \stackrel{\leftrightarrow}{\rightarrow} \mathbb{Z}^{2}(\pi, \mathbb{R})
$$

induces the a skew-symmetric pairing on cohomology

$$
H^{1}\left(\pi, \mathfrak{S}_{\rho}\right) \times H^{1}\left(\pi, \mathfrak{H}_{\rho}\right) \rightarrow H^{2}(\pi, \mathbb{R}) \simeq \mathbb{R} .
$$

This skew-symmetric pairing induces the symplectic structure on $\mathcal{M}$.

We assume henceforth that $\rho$ is irreducible. For simplicity we write $x \cdot u$ $(y)$ or just $x u(y)$ for $\operatorname{Ad}_{\rho}(x)(u(y))$. We know from $[1, \S(3 \cdot 4)]$ that the following formula

$$
\omega(u, v)=-\sum_{i=1}^{g}\left\{\left\langle u\left(\# \frac{\partial R}{\partial A_{\imath}}\right), v\left(A_{\imath}\right)\right\rangle+\left\langle u\left(\# \frac{\partial R}{\partial B_{\imath}}\right), v\left(B_{\imath}\right)\right\rangle\right\}
$$

(for $\left.u, v \in Z^{1}\left(\pi, \mathfrak{H}_{\rho}\right)\right)$ gives the symplectic form on $H^{1}\left(\pi, \mathfrak{S}_{\rho}\right)$.

\section{§2. Mutually Dual Presentations of the Fundamental Group}

If $\pi=\left\langle A_{1}, B_{1}, \ldots, A_{g}, B_{g} \mid \prod_{\imath=1}^{g}\left[A_{\imath}, B_{l}\right]=I\right\rangle$ is a given presentation then we set

$$
\begin{aligned}
& \alpha_{\imath}=C_{\imath-1} B_{\imath}^{-1} C_{l}^{-1} \\
& \beta_{\imath}=C_{\imath} A_{\imath}^{-1} C_{1-1}^{-1}
\end{aligned}
$$

for $i=1, \ldots, g$ where $C_{i}=\prod_{j=1}^{t}\left[A_{3}, B_{\jmath}\right]$. It can be checked that $\prod_{i=1}^{g}\left[\alpha_{\imath}, \beta_{l}\right]=I$ so that we have another presentation

$$
\pi=\left\langle\alpha_{1}, \beta_{1}, \ldots, \alpha_{g}, \beta_{g} \mid \prod_{l=1}^{g}\left[\alpha_{i}, \beta_{l}\right]=I\right\rangle .
$$

If we set $\mathscr{C}_{l}=\prod_{j=1}^{i}\left[\alpha_{j}, \beta_{j}\right]$. then it can be checked that

$$
\begin{aligned}
& A_{\imath}=\mathscr{C}_{1-1} \beta_{l}^{-1} \mathscr{C}_{\imath}^{-1} \\
& B_{l}=\mathscr{C}_{\imath} \alpha_{l}^{-1} \mathscr{C}_{t-1}^{-1}
\end{aligned}
$$

for $i=1, \ldots, g$ where $\mathscr{C}_{i}=\prod_{j=1}^{i}\left[\alpha_{j}, \beta\right]$.

Consequently we see that both the above presentations of $\pi$ are 'dual' to each other. 


\section{§3. An Inner Product on $\mathbb{Z}^{1}\left(\pi, \mathfrak{S}_{o}\right)$.}

Given a presentation $\pi=\left\langle A_{1}, B_{1}, \ldots, A_{g}, B_{g} \mid \mathbb{I}\left[A_{\imath}, B_{\imath}\right]=1\right\rangle$, define the symmetric pairing on $Z^{1}\left(\pi, \mathfrak{S}_{\rho}\right)$ by

$$
\langle u, v\rangle_{o}=\sum_{i=1}^{g}\left\langle u\left(\alpha_{i}\right), v\left(\alpha_{i}\right)\right\rangle+\left\langle u\left(\beta_{i}\right), v\left(\beta_{i}\right)\right\rangle
$$

where $\alpha_{i}, \beta_{\imath}$ are the dual generators defined in $\S 2 .\langle\rangle_{\rho}$ defines an inner product on $Z^{1}\left(\pi, \mathfrak{S}_{\rho}\right)$, since for $u \in Z^{1}\left(\pi, \mathfrak{S}_{\rho}\right)$

$$
\begin{aligned}
\langle u, u\rangle_{\rho}=0 & \Rightarrow\left\langle u\left(\alpha_{\imath}\right), u\left(\alpha_{\imath}\right)\right\rangle=0=\left\langle u\left(\beta_{\imath}\right), u\left(\beta_{\imath}\right)\right\rangle \text { for } i=1, \ldots, g . \\
& \left.\Rightarrow u\left(\alpha_{\imath}\right)=u\left(\beta_{\imath}\right)\right\rangle=0 \text { since }\langle,\rangle \text { is an inner product on } \mathfrak{S} \\
& \Rightarrow u \equiv 0 \text { since } u \text { vanishes on the generators } \alpha_{\imath}, \beta_{\imath} \text { of } \pi .
\end{aligned}
$$

For $\mu \in \mathfrak{F}_{\rho}$ its co-boundary $\partial \mu: \pi \longrightarrow \mathfrak{S}_{\rho}$ is defined by $(\partial \mu)(x)=x \cdot \mu-\mu$ for $x$ $\in \pi$. The space $B^{1}\left(\pi, \mathfrak{S}_{\rho}\right)$ is the space of coboundaries. Using the inner-product \langle\rangle$_{\rho}$ on $Z^{1}\left(\pi, \mathfrak{S}_{\rho}\right)$ we can write

$$
Z^{1}\left(\pi, \mathfrak{S}_{\rho}\right)=B^{1}\left(\pi, \mathfrak{H}_{\rho}\right) \oplus B^{1}\left(\pi, \mathfrak{S}_{\rho}\right)^{\perp} .
$$

As in [2], we call $B^{1}\left(\pi, \mathfrak{S}_{\rho}\right)^{\perp}$ the space of 'harmonic' cocycles.

$$
B^{1}\left(\pi, \mathfrak{H}_{\rho}\right)^{\perp}=\left\{u \in Z^{1}\left(\pi, \mathfrak{H}_{\rho}\right) \mid\langle u, \partial \mu\rangle_{\rho}=0 \forall \mu \in \mathfrak{H}_{\rho}\right\} .
$$

Now $\langle u, \partial \mu\rangle_{\rho}$

$$
\begin{aligned}
& =\sum_{i=1}^{g}\left\{\left\langle u\left(\alpha_{i}\right), \partial \mu\left(\alpha_{\imath}\right)\right\rangle+\left\langle u\left(\beta_{i}\right), \partial \mu\left(\beta_{\imath}\right)\right\rangle\right\} \\
& =\sum_{i=1}^{g}\left\{\left\langle u\left(\alpha_{\imath}\right), \alpha_{\imath} \cdot \mu-\mu\right\rangle+\left\langle u\left(\beta_{\imath}\right), \beta_{i} \cdot \mu-\mu\right\rangle\right\} \\
& =\sum_{i=1}^{g}\left\{\left\langle\alpha_{\imath}^{-1} \cdot u\left(\alpha_{\imath}\right), \mu\right\rangle-\left\langle u\left(\alpha_{\imath}\right), \mu\right\rangle+\left\langle\beta_{\imath}^{-1} \cdot u\left(\beta_{\imath}\right), \mu\right\rangle-\left\langle u\left(\beta_{\imath}\right), \mu\right\rangle\right\} \\
& =-\sum_{\imath=1}^{g}\left\langle u\left(\alpha_{\imath}^{-1}\right)+u\left(\alpha_{\imath}\right)+u\left(\beta_{\imath}^{-1}\right)+u\left(\beta_{\imath}\right), \mu\right\rangle \\
& \text { since } \alpha_{i}^{-1} \cdot u\left(\alpha_{i}\right)=-u\left(\alpha_{i}^{-1}\right) \\
& \beta_{l}^{-1} \circ u\left(\beta_{\imath}\right)=-u\left(\beta_{l}^{-1}\right) \\
& \langle u, \partial \mu\rangle_{\rho}=0 \forall \mu \Rightarrow\left\langle\sum_{i=1}^{g}\left\{u\left(\alpha_{\imath}\right)+u\left(\alpha_{i}^{-1}\right)+u\left(\beta_{\imath}\right)+u\left(\beta_{i}^{-1}\right)\right\}, \mu\right\rangle=0 \\
& \Rightarrow \sum_{i=1}^{g}\left\{u\left(\alpha_{i}\right)+u\left(\alpha_{\imath}^{-1}\right)+u\left(\beta_{\imath}\right)+u\left(\beta_{\imath}^{-1}\right)\right\}=0
\end{aligned}
$$

since $\langle$, $\rangle$ is non-degenerate on $\mathfrak{S}_{\rho}$.

Thus we have the following characterisation of harmonic cocycles.

$$
B^{1}\left(\pi, \mathfrak{S}_{\rho}\right)^{\perp}=\left\{u \in Z^{1}\left(\pi, \mathfrak{S}_{\rho}\right) \mid \sum_{i=1}^{g}\left\{u\left(\alpha_{i}\right)+u\left(\alpha_{i}^{-1}\right)+u\left(\beta_{\imath}\right)+u\left(\beta_{\imath}^{-1}\right)\right\}=0\right\}
$$




\section{§4. The Symplectic Pairing and Its Relationship to Mutually Dual Generators}

For $u \in Z^{1}\left(\pi, \mathfrak{S}_{\rho}\right)$, let $u^{\perp}$ be its harmonic part i.e $u^{\perp}$ is the projection of $u$ onto $B^{1}\left(\pi, \mathfrak{S}_{\rho}\right)^{\perp}$. As in $[2, \S 1]$, we define the map $\phi: Z^{1}\left(\pi, \mathfrak{S}_{\rho}\right) \rightarrow Z^{1}\left(\pi, \mathfrak{S}_{\rho}\right)$ as

$$
\begin{aligned}
& \phi(u)\left(A_{\imath}\right)=u^{\perp}\left(\# \frac{\partial R}{\partial A_{\imath}}\right) \\
& \phi(u)\left(B_{i}\right)=u^{\perp}\left(\# \frac{\partial R}{\partial B_{\imath}}\right) \text { for } i=1, \ldots, g .
\end{aligned}
$$

We need to check that $\phi(u)$ is a 1 -cocycle or equivalently $[1, \S 3 \cdot 6]$ that the following identity holds

$$
\phi(u)(R)=\sum_{\imath=1}^{g}\left\{\frac{\partial R}{\partial A_{\imath}} \phi(u)\left(A_{\imath}\right)+\frac{\partial R}{\partial B_{\imath}} \phi(u)\left(B_{\imath}\right)\right\}=0 .
$$

Now $\frac{\partial R}{\partial A_{\imath}} \phi(u)\left(A_{\imath}\right)$

$$
\begin{aligned}
& =\frac{\partial R}{\partial A_{\imath}} u^{\perp}\left(\# \frac{\partial R}{\partial A_{\imath}}\right) \\
& =\left(C_{i}-C_{\imath} B_{i}\right) u^{\perp}\left(C_{\imath-1}^{-1}-B_{i}^{-1} C_{\imath}^{-1}\right) \\
& =C_{\imath} u^{\perp}\left(C_{\imath-1}^{-1}\right)-C_{\imath} B_{\imath} u^{\perp}\left(C_{\imath-1}^{-1}\right)-C_{\imath-1} u^{\perp}\left(B_{\imath}^{-1} C_{\imath}^{-1}\right)+C_{\imath} B_{\imath} u^{\perp}\left(B_{\imath}^{-1} C_{\imath}^{-1}\right) \\
& =-\left\{u^{\perp}\left(C_{\imath-1}\right)+C_{1-1} u^{\perp}\left(B_{\imath}^{-1} C_{i}^{-1}\right)\right\}-\left\{C_{\imath} B_{i} u^{\perp}\left(C_{\imath-1}^{-1}\right)+u\left(C_{\imath} B_{\imath}\right)\right\} \\
& =-\left\{u^{\perp}\left(C_{i-1} B_{\imath}^{-1} C_{\imath}^{-1}\right)+u\left(C_{\imath} B_{\imath} C_{\imath-1}^{-1}\right)\right\} \\
& =-\left\{u^{\perp}\left(\alpha_{\imath}\right)+u^{\perp}\left(\alpha_{\imath}^{-1}\right)\right\} .
\end{aligned}
$$

Similarly

$$
\begin{aligned}
\frac{\partial R}{\partial B_{\imath}} \phi(u)\left(B_{\imath}\right) & =\frac{\partial R}{\partial B_{\imath}} u^{\perp}\left(\# \frac{\partial R}{\partial B_{\imath}}\right) \\
& =\left(C_{\imath} A_{\imath}-C_{\imath}\right) u^{\perp}\left(A_{i}^{-1} C_{\imath-1}^{-1}-C_{\imath}^{-1}\right) \\
& =-u^{\perp}\left(C_{\imath-1} A_{\imath}\right)-C_{\imath} u^{\perp}\left(A_{\imath}^{-1} C_{\imath-1}^{-1}\right)-C_{\imath-1} A_{\imath} u^{\perp}\left(C_{\imath}^{-1}\right)-u^{\perp}\left(C_{\imath}\right) \\
& =-\left\{u^{\perp}\left(C_{\imath-1} A_{\imath} C_{\imath}^{-1}\right)+u^{\perp}\left(C_{\imath} A_{\imath}^{-1} C_{\imath-1}^{-1}\right)\right\} \\
& =-\left\{u^{\perp}\left(\beta_{i}\right)+u^{\perp}\left(\beta_{\imath}^{-1}\right)\right\} .
\end{aligned}
$$

Thus

$$
\begin{aligned}
& \sum_{i=1}^{g}\left\{\frac{\partial R}{\partial B_{\imath}} \phi(u)\left(A_{\imath}\right)+\frac{\partial R}{\partial B_{\imath}} \phi(u)\left(B_{\imath}\right)\right\} \\
= & -\sum_{i=1}^{g}\left\{u^{\perp}\left(\alpha_{\imath}\right)+u^{\perp}\left(\alpha_{i}^{-1}\right)+u^{\perp}\left(\beta_{\imath}\right)+u^{\perp}\left(\beta_{i}^{-1}\right)\right\} \\
= & 0
\end{aligned}
$$


since $u^{\perp}$ is a harmonic cocycle.

Consequently $\phi: Z^{1}\left(\pi, \mathfrak{S}_{\rho}\right) \longrightarrow Z^{1}\left(\pi, \mathfrak{H}_{\rho}\right)$ is well defined. We can identify $B^{1}\left(\pi, \mathfrak{S}_{\rho}\right)^{\perp}$ with $H^{1}\left(\pi, \mathfrak{S}_{\rho}\right)$. Now for harmonic cocycles $u, v \in B^{1}\left(\pi, \mathfrak{S}_{\rho}\right)^{\perp} \approx H^{1}(\pi$, $\mathfrak{S}_{\rho}$ ) (i.e. $u=u^{\perp}, v=v^{\perp}$ ) we check the following identity

$$
\omega(u, \phi(v))=-\langle u, v\rangle_{\rho} .
$$

We know from $\S 1$ that

$$
\omega(u, \phi(v))=-\sum_{i=1}^{g}\left\{\left\langle u\left(\# \frac{\partial R}{\partial A_{\imath}}\right), \phi(v)\left(A_{\imath}\right)\right\rangle+\left\langle u\left(\# \frac{\partial R}{\partial B_{\imath}}\right), \phi(v)\left(B_{\imath}\right)\right\rangle\right\} .
$$

It follows from the definition of dual generators that

$$
\# \frac{\partial R}{\partial A_{\imath}}=C_{\imath-1}^{-1}-B_{\imath}^{-1} C_{\imath}^{-1}=C_{\imath-1}^{-1}-C_{\imath-1}^{-1} \alpha_{\imath}
$$

so that

$$
\begin{aligned}
u\left(\# \frac{\partial R}{\partial A_{\imath}}\right) & =u\left(C_{\imath-1}^{-1}\right)-u\left(C_{t-1}^{-1} \alpha_{\imath}\right) \\
& =u\left(C_{\imath-1}^{-1}\right)-u\left(C_{\imath-1}^{-1}\right)-C_{\imath-1}^{-1} u\left(\alpha_{\imath}\right) \\
& =-C_{\imath-1}^{-1} u\left(\alpha_{\imath}\right) .
\end{aligned}
$$

Similarly

$$
\# \frac{\partial R}{\partial B_{t}}=A_{\imath}^{-1} C_{t-1}^{-1}-C_{\imath}^{-1}=C_{t}^{-1} \beta_{\imath}-C_{t}^{-1}
$$

so that

$$
\begin{aligned}
u\left(\# \frac{\partial R}{\partial B_{\imath}}\right) & =u\left(C_{\imath}^{-1} \beta_{\imath}-C_{\imath}^{-1}\right)-u\left(C_{\imath}^{-1}\right) \\
& =u\left(C_{\imath}^{-1}\right)-C_{\imath}^{-1} u\left(\beta_{\imath}\right)-u\left(C_{\imath}^{-1}\right) \\
& =C_{\imath}^{-1} u\left(\beta_{\imath}\right) .
\end{aligned}
$$

We therefore have

$$
\begin{aligned}
\left\langle u\left(\# \frac{\partial R}{\partial A_{\imath}}\right), \phi\left(v^{\prime}\right)\left(A_{\imath}\right)\right\rangle & =\left\langle u\left(\# \frac{\partial R}{\partial A_{\imath}}\right), v\left(\# \frac{\partial R}{\partial H_{\imath}}\right)\right\rangle \text { since } v=v^{\perp} \\
& =\left\langle-C_{t-1}^{-1} u\left(\alpha_{\imath}\right),-C_{\imath-1}^{-1} \imath^{\prime}\left(\alpha_{\imath}\right)\right\rangle \\
& =\left\langle u\left(\alpha_{\imath}\right), v\left(\alpha_{\imath}\right)\right\rangle .
\end{aligned}
$$

Similary

$$
\left\langle u\left(\# \frac{\partial R}{\partial B_{\imath}}\right), \phi(v)\left(B_{\imath}\right)\right\rangle=\left\langle u\left(\beta_{t}\right), v\left(\beta_{\imath}\right)\right\rangle .
$$

Thus we have 


$$
\begin{aligned}
\omega(u, \phi(v)) & =-\sum_{i=1}^{g}\left\{\left\langle u\left(\alpha_{\imath}\right), v\left(\alpha_{\imath}\right)\right\rangle+\left\langle u\left(\beta_{t}\right), v\left(\beta_{\imath}\right)\right\rangle\right\} \\
& =-\langle u, v\rangle_{\rho} .
\end{aligned}
$$

The identity $\omega(u, \phi(v))=-\langle u, v\rangle_{\rho}$ suggests that $\phi$ may induce a complex structure on $B^{1}\left(\pi, \mathfrak{H}_{\rho}\right)^{\perp} \approx H^{1}\left(\pi, \mathfrak{H}_{\rho}\right)$ i.e. $\phi^{2}=-I d$ on $H^{1}\left(\pi, \mathfrak{S}_{\rho}\right)$ and is integrable. But we are unable to prove it. We leave it as a conjecture. Thus the symplectic structure $\omega$ on $M=\operatorname{Hom}(\pi, G) / G$ intertwines the mutually dual presentations of $\pi$. The geometry behind this intertwining phenomenon is yet to be explored.

\section{References}

[1] Goldman. W, The symplectic nature of fundamental groups of surfaces. Adv. $n$. Math., 54 (1981), 200-2:25.

[2] Guruprasad. K. and Rajan, C.S.. Group cohomology and the symplectic structure on the moduli space of representations, to appear in the Dukf Math $J$.

[3] Saito, K. Isıng model on Fuchsian group, preprint. 
